\title{
¿DERECHOS HUMANOS O DERECHOS LIBERALES?
}

José Rubio-Carracedo

Universidad de Málaga (España)

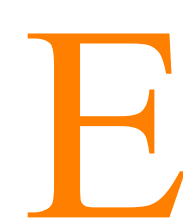

1 alcance y la significación de los derechos humanos sigue siendo materia controvertida. Significan, sin duda, la aportación más valiosa de Occidente a la humanidad. Pero, ¿hasta qué punto son aplicables al conjunto de los países fuera de los que comparten el régimen democrático? Y, en el caso de que la respuesta sea positiva, ¿no será precisa una laboriosa, y siempre problemática, traducción e interpretación de los mismos a las categorías socioculturales de cada país? Porque lo cierto es que el origen de los derechos humanos en Occidente puede remontarse, al menos, hasta el cristianismo, pero su formulación actual y su vigencia procede de las revoluciones liberales -esto es, burguesas- del siglo XVIII. Es más, durante el siglo XIX y principios del $\mathrm{XX}$, la doctrina de los derechos humanos quedó oscurecida tras los pliegues del estatalismo, y posteriormente burlada en los regímenes totalitarios; sólo tras la segunda guerra mundial, que significó el triunfo genérico de los regímenes democráticos sobre los regímenes totalitarios, con la solemne Declaración Universal de 1948, se ha iniciado la era efectiva de los derechos humanos en occidente y los primeros intentos para su universalización. En definitiva, para que los derechos humanos se hagan efectivamente derechos civiles y políticos jurídicamente reconocidos.

Ahora bien, lo menos que se puede decir es que tales intentos de universalización, por lo general en el marco institucional de las Naciones Unidas, han sido tan torpes como poco efectivos. Porque se ha pretendido universalizar no sólo el espíritu sino también la letra occidental de los derechos humanos. Ello ha significado, en la práctica, un intento de universalizar, conjuntamente con los derechos humanos, las categorías e instituciones del liberalismo en todo el planeta, esto es, la «occidentalización» del mundo. Tal intento no sólo ha fracasado, sino que ha provocado los reproches de imperialismo cultural y de capitalismo etnocéntrico y, lo que es peor, ha provocado la confusión y el prejuicio generalizado de que los derechos huma- 
nos también son solamente categorías y conceptos del liberalismo occidental, que sólo en su ámbito encuentran sentido, y que no resulta legítimo extender fuera de sus fronteras socioculturales.

El clima de confusión ha alcanzado también a Occidente, sobre todo con la eclosión de la postmodernidad en Europa y del comunitarismo en los Estados Unidos. Hasta el punto de que ha llegado a ponerse de moda la consideración de que los derechos humanos son meramente derechos liberales, ya que se corresponden con una mentalidad individualista y una concepción atomista de la sociedad propias y características del liberalismo occidental. No resultaría, pues, legítimo el intento de universalizarlos ni siquiera vía traducción e interpretación sociocultural. Esta situación de asedio interno a los derechos humanos ha condicionado, a su vez, propuestas minimalistas o rebajadas en los autores que todavía mantienen la significación y el alcance universalista de los valores humanos en sí mismos, independientemente del ropaje o la envoltura liberal. Probablemente estos intentos, por insatisfactorios que resulten todavía, marcan el rumbo a seguir en el futuro: los derechos humanos tienen un alcance y una significación universal pero con la condición fundamental de que se desprendan de la letra liberal para que su espíritu pueda ser traducido e interpretado en las categorías y valores de cada cultura, ofreciendo así la posibilidad de que efectúen en tales países una revolución humanista semejante a la que provocaron en Occidente. Obviamente, tal proceso de transculturación resulta sumamente difícil de realizar desde las categorías liberales vigentes; se precisa más bien un laborioso esfuerzo cooperativo, tanto intelectual como institucional, en el que resultará decisiva la participación de los mismos destinatarios.

\section{a) los derechos humanos como derechos aristocráticos para todos los ciudadanos}

En un texto reciente, M. Walzer ${ }^{1}$ ha apuntado la idea de que el principio fundamental que rige la esfera política, esto es, que «el poder únicamente puede ser obtenido y mantenido con el consentimiento de los gobernados», es anterior a la democracia moderna, pues ya estaba vigente en la monarquía medieval, aunque el consenso de los gobernados se limitaba unos pocos, a la nobleza. La esencia de las revoluciones democráticas consistió, a su juicio, en reemplazar a esos «pocos» por «todos» los gobernados. Por lo mismo, el ideal del ciudadano moderno se moldeó sobre el modelo aristocrático, al tomar del mismo los derechos que prestan significación a su consentimiento, así como el elenco de actividades y el espacio social. Consiguientemente -añado yo- los derechos humanos se moldearon

${ }^{1}$ M. Walzer, Moralidad en el ámbito local e internacional. Madrid, Alianza, 1996, 85ss. 
igualmente sobre los derechos aristocráticos, los que los nobles siempre tuvieron: derecho de petición, de reunirse en asamblea, de expresión, de conciencia (religiosa) y hasta de privacidad (siguiendo la máxima «mi casa es mi castillo»).

En efecto, cuando se estudian desde esta óptica las Declaraciones de Derechos revolucionarias se percibe claramente el celo burgués-ciudadano por enumerar exhaustivamente todos los derechos de la nobleza para no renunciar ni a uno sólo de los mismos. El ejemplo más claro lo ofrece, sin duda, el Bill of Rights de 1689 con el que culmina la «Revolución Gloriosa» británica y el establecimiento de la primera monarquía parlamentaria moderna. Pero lo más llamativo es que, al menos en lo que respecta a garantizar las libertades, el Bill of Rights de 1689 enlaza histórica y doctrinalmente con la Magna Charta de 1215, con la Petition of Right de 1628 y el Habeas Corpus Act de 1679. Como ha hecho notar M. Fioravanti ${ }^{2}$, la Magna Charta no puede confundirse con los contratos simples de dominación que se pactaban por doquier en la Europa Medieval entre el monarca y la nobleza, en los que se establecían las condiciones y límites de la obediencia debida. Porque, aparte de la «libertad personal» típica de los tratados de dominación, la Magna Charta pone un énfasis especial en la «libertad como seguridad personal y de la propiedad». Así el art. 39 establece: «Ningún hombre podrá ser detenido o encarcelado, o privado de sus derechos o de sus bienes, o puesto fuera de la ley o exiliado, o privado de su rango de cualquier otro modo, ni usaremos de la fuerza contra él, o enviaremos a otros para que lo hagan, excepto por sentencia judicial de sus pares y según la ley del país». Ciertamente, las exigencias de libertad individual y de juicio entre pares se refieren primordialmente al estamento aristocrático; pero las garantías que se exigen sobre la libertad como seguridad jurídica y de los propios bienes, y especialmente la exigencia de una «sentencia judicial por sus pares y según la ley del país» significa un paso fundamental para quebrar el arbitrismo del monarca y someterlo a la jurisprudencia de tal modo que se pasa casi insensiblemente de la «ley del país»al «derecho común» (common law). No sólo ha nacido el tercer poder del estado, el judicial, sino también el estatuto de las libertades ciudadanas. El enfoque historicista dominante en la tradición británica impidió, por una parte, que el despotismo real llegase a ser nunca tan desmesurado como en el resto del continente europeo y, por otra, facilitó que los derechos nobiliarios se fueran extendiendo progresivamente a la gentry y a la burguesía comercial casi sin solución de continuidad. El Bill of Rights de 1689 com-

${ }^{2}$ M. Fioravanti, Los derechos fundamentales. Apuntes de historia de las constituciones. Madrid, Trotta, 1996, 31 ss. 
pletará el proceso no sólo al poner el énfasis en la soberanía parlamentaria (King in Parliament), sino también al reforzar definitivamente la mayoría de edad de los ciudadanos materializada en la capacidad que les reconoce (derechos y libertades) no sólo para elegir libremente a los miembros del parlamento (art. 8) sino también para ejercer derechos típicos de la aristocracia como el de presentar peticiones al rey sin temor a ser castigados por ello (art. 5), así como la obligatoriedad de que toda multa o confiscación sea precedida de sentencia judicial (art. 12). Hasta tal punto que el proceso revolucionario que culminó con la independencia de los Estados Unidos se inició por la protesta contra los abusos fiscales de la metrópoli y su incumplimiento del principio no taxation without representation. En definitiva, no sólo surge el primer esbozo de los derechos humanos, sino que tales derechos nacen ya positivados y protegidos jurídicamente, esto es, en cuanto derechos civiles y derechos políticos, por muy restringido que fuese el sujeto de tales derechos.

Las Declaraciones de derechos subsiguientes a las Revoluciones Americana y Francesa persiguen el mismo objetivo, pero por una vía diametralmente opuesta: rechazan el modelo historicista-continuista británico justamente para marcar las diferencias del «Nuevo» frente al «Antiguo Régimen», que pretenden abolir desde sus cimientos, desde un enfoque racionalista. No pudieron evitar, sin embargo, que su modelo «individualista», pese a su negación radical del «orden jurídico estamental», reconstruyera reactivamente de modo racional y abstracto (iusnaturalista y contractualista) los derechos «del hombre y del ciudadano» sobre el molde histórico del aristócrata, ahora generalizado a todos los hombres libres, con el objetivo primordial de garantizar los derechos y libertades frente a toda veleidad arbitraria de los poderes estatales. De aquí que el énfasis se pusiera aquí en el punto de vista moral y político. Resulta muy significativo en esta línea el proceder de los «padres fundadores» de la Revolución Americana quienes, tras haber elaborado el texto constitucional de 1787 según el modelo individualista racional y abstracto, sólo dos años después, a iniciativa de Madison, creyeron indispensable para facilitar su positivación añadirle un Bill of Rights siguiendo el modelo historicista británico, que entró en vigor bajo la forma de las diez primeras enmiendas de la Constitución Americana en 1791, y que confirman fehacientemente cómo en un país sin aristocracia de sangre todos los ciudadanos pasaban a disfrutar en cuanto derecho común de los antiguos privilegios de la nobleza. Y, en la misma línea, asistimos hoy a un intento paralelo -todavía ciertamente muy ambiguopara universalizar a los ciudadanos del mundo los derechos fundamentales que les son reconocidos a los ciudadanos de las democracias liberales, al menos en versiones minimalistas de los mismos. 


\section{b) John Rawls y la universalización de los derechos humanos: maximalismo en la justificación}

En este trabajo, no obstante, me limitaré a examinar uno de los intentos minimalistas que más eco ha encontrado, el de John Rawls en su escrito The Law of Peoples ${ }^{3}$, donde amplía el ámbito de aplicación de los derechos humanos a las sociedades jerárquicas, además de las sociedades democráticas de Occidente. Para ello parte de una concepción minimalista de los derechos humanos, de modo que tales derechos no dependen de ninguna doctrina moral particular (comprehensiva) ni de ninguna concepción filosófica de la naturaleza humana (ni siquiera la liberal), si no que «expresan el patrón mínimo de instituciones políticas bien-ordenadas para todos los pueblos» que constituyen de este modo «una sociedad política justa de pueblos». Toda violación sistemática de tales derechos significaría un problema serio para el conjunto de tal «sociedad de pueblos, tanto los liberales como los jerárquicos» ${ }^{4}$.

Y ¿qué entiende Rawls por «sociedad jerárquica»? Desde luego, no son sociedades liberales, aunque sí son capaces de participar en «una posición original con velo de ignorancia» en cuanto artificio de representación para decidir la adopción del «derecho de los pueblos», en la teoría ideal de la justicia, al igual que las sociedades liberales bien-ordenadas. Rawls señala tres requisitos para toda sociedad jerárquica bien-ordenada: $1^{\circ}$, ha de ser pacífica, de modo que persiga sus objetivos mediante la diplomacia y el comercio, u otros medios pacíficos. De donde se sigue que la doctrina religiosa, que impregna su política, ha de ser no-expansionista y respetar el orden civil y la integridad, independencia y libertades de las demás sociedades; $2^{\circ}$, su sistema de derecho no es meramente coercitivo, sino que impone obligaciones moralmente vinculantes a sus miembros; esto es, su sistema de derecho se basa sobre una creencia sincera y razonable «de guiarse por una concepción común de la justicia». Ello implica que tal sociedad está dotada de una razonable «jerarquía consultiva», esto es, de cuerpos o asambleas representativas, cuyo cometido es velar por los intereses fundamentales de todos los miembros de la sociedad. Obviamente en tales cuerpos o asambleas representativas se da pluralismo y posibilidad de disenso, de modo que los jueces y los funcionarios han de tomarlos en serio; $3^{\circ}$, por último, tal socie-

${ }^{3}$ J. Rawls, The Law of Peoples, en S. Shute \& S. Hurley, eds., On Human Rights. The Oxford Anmesty Lectures 1993. New York, Basic Books, 1993, 41-82 (sigla LP). Una versión más amplia y detallada de este trabajo aparecerá en el $n^{0}$ monográfico que Daimon dedica a los 25 años de A Theory of Justice, con el título de «La teoría rawlsiana de la justicia internacional: maximalismo en la justificación, minimalismo en la aplicación».

${ }^{4}$ LP 68. 
dad respeta «los derechos humanos básicos», esto es, asegura a todos sus miembros «ciertos derechos mínimos» de subsistencia y seguridad (derecho a la vida), de libertad, de propiedad personal, así como la «igualdad formal» que viene expresada por «las reglas de la justicia natural» en el sentido de Hart ${ }^{5}$. Tal sociedad ha de garantizar igualmente la «tolerancia religiosa», puesto que «admite una medida» de libertad de conciencia y de pensamiento, aun cuando tal libertad no sea tan general e igual como en las sociedades liberales, y aunque tenga una religión oficial con ciertos privilegios. Y Rawls aclara que no se trata de que se acerquen lo más posible al credo liberal, sino que se trata de que sean «razonables» dentro de su propia concepción de la justicia ${ }^{6}$.

Es de notar que Rawls no parte de datos ni de referencias concretas, del mismo modo que no precisa apenas los caracteres generales de tales sociedades jerárquicas realmente existentes, fuera de su énfasis sobre los fundamentos religioso-filosóficos de tales estados. Él se atiene, como siempre, a su metodología constructivista kantiana. Pero no parece ser consciente de que la aplicación de tal método a sociedades no liberales conlleva nuevos problemas y difícilmente resolubles. En efecto, el constructivismo kantiano parte siempre de unas convicciones liberales compartidas que procede a «construir» válidamente en la posición original con el velo de ignorancia, por lo que su aplicación a las sociedades jerárquicas no liberales ha de hacerse en base a meras analogías, ya que no comparten las convicciones liberales.

Pero lo decisivo es que Rawls se propone ampliar el ámbito de aplicación de los derechos humanos liberales mediante dos procedimientos: a) asimilación metodológica de ciertos tipos de sociedades no liberales que denomina «jerárquicas» a los valores y modos de actuar de las sociedades liberales o modernas democracias occidentales; y b) exigencia minimalista (un «mínimo», de derechos humanos «básicos», una «medida» de los derechos fundamentales de libertad de pensamiento y de expresión) de los derechos humanos tal como vienen formulados en las solemnes declaraciones occidentales, lo que implica una selección de los mismos en cuanto más fundamentales o menos. En el primer procedimiento Rawls se muestra innecesariamente maximalista; en segundo, arriesgadamente minimalista.

Respecto del primer procedimiento, parece tan inadecuada como innecesaria la asimilación metodológica de aplicar a ambos tipos de sociedad -liberal y jerárquica- el mismo «artificio de representación» de la posición original. En efecto, la metodología constructivista por su propia naturaleza sólo

\footnotetext{
${ }^{5}$ LP 60-63.

${ }^{6}$ LP 63-64.
} 
puede validar a nivel normativo-reflexivo (la posición original) unas convicciones o tradiciones firmes y compartidas a nivel histórico-empírico. En el caso de Rawls, lo que somete a la prueba del constructivismo kantiano es la tradición liberal tal como se expresa en el concepto ampliamente compartido en las democracias liberales de la cooperación social, en cuanto que consta de dos partes íntimamente entrelazadas: el rational (los beneficios racionales de la cooperación o racionalidad estratégica) y el reasonable (las condiciones equitativas e imparciales que aseguran la cooperación) $)^{7}$. Entonces carece de sentido someter al constructivismo kantiano las convicciones y tradiciones compartidas de las sociedades jerárquicas; o, más exactamente, carece de sentido pretender que la tradición jerárquica, sometida al procedimiento reflexivo-validante de la posición original, pueda decantarse por los derechos humanos, que son extraños e incompatibles con la misma tanto como se contienen implícitamente en la cultura pública de una sociedad democrática.

Lo más llamativo es que Rawls no apele aquí a su teoría del «consenso entreverado»

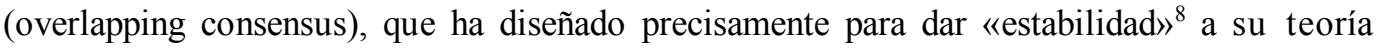
de la justicia como equidad en cuanto teoría política. Rawls, en efecto, asume que su teoría confrontada con otras rivales (aunque cite como «caso modelo» la confrontación con la concepción religiosa cristiana, la concepción filosófica kantiana, la utilitarista y la concepción pluralista) ${ }^{9}$ logra generar un consenso en buena medida (aunque siempre parcialmente) coincidente, de tal modo que todas las teorías confrontadas terminan por entreverarse (y converger, en definitiva, sobre su teoría).

Pero el alcance de este consenso entreverado no es ilimitado; Rawls lo plantea siempre dentro del ámbito occidental y es lógico que sea así ya que, en definitiva, su teoría liberal persigue obtener el respaldo de un consenso entreverado; es decir, de un consenso a través del cual llegue a ser aceptada por todas las doctrinas morales, religiosas y filosóficas rivales, que se han ido desarrollando durante generaciones en un estado más o menos justo y democrático, cuyo criterio de justicia es precisamente esta concepción política. Pero si se muestra dispuesto a dar un paso tan desproporcionado como el de llevar las doctrinas religiosas o filosóficas de las sociedades jerárquicas a la «posición original», mucho más sencillo y proporcionado hubiera sido tender un puente a tales sociedades jerárquicas mediante su metodología del «consenso entreverado», aunque nunca aplicado de modo simple y directo, sino mediante un diálogo transcultural, con las consiguientes traducciones e interpretaciones interculturales.

\footnotetext{
${ }^{7}$ J. Rawls, Political Liberalism. New York, Columbia University Press, 48-54. (sigla PL).

${ }^{8}$ PL XV-XVI.

${ }^{9}$ PL 145ss.
} 
En efecto, este consenso entreverado obtenido mediante diálogo y transposición intercultural de conceptos, no sólo parece más apropiado y accesible, sino que evita el maximalismo de Rawls al pretender obtener el mismo objetivo mediante la aplicación simple y directa del artificio de representación que es la posición original, porque al tratarse de una metodología constructiva solamente podrá validar reflexiva y cuasitrascendentalmente las convicciones más fuertes y compartidas de las sociedades jerárquicas que, por definición, son diferentes a las que sostienen a las sociedades democráticas liberales. Rawls, en cambio, parece atribuir ahora a la posición original una capacidad totalmente irreal de poder actuar como crisol intercultural, cuyo fruto sería un consenso directo y simple sobre los derechos humanos más básicos, siendo así que para el diálogo de su teoría política de la justicia con las doctrinas morales comprehensivas vigentes en las sociedades democráticas occidentales -y, por tanto, mucho más próximas- exige la metodología del consenso entreverado que, además, resulta justificado aplicar, no sobre cualquier pluralismo, sino específicamente sobre el «pluralismo razonable» en cuanto que es fruto «del libre ejercicio de la razón libre humana en condiciones de libertad» ${ }^{10}$.

Tanto más cuanto que, en realidad, el consenso entreverado tiene un diseño asimilacionista, como se infiere de las respuestas que da Rawls a las objeciones que le han sido formuladas. En efecto, el consenso entreverado no significa una mera negociación ni un simple modus vivendi, pues la concepción pública de la justicia que representa su teoría es fruto de la posición original, por lo que «el objeto del acuerdo, la concepción política de la justicia, es él mismo una concepción moral/.../ y esta concepción es también asumida por motivos morales $/ \ldots /\rangle^{11}$. Son las demás doctrinas comprehensivas las que deben acercarse hasta entreverarse con la concepción liberal de la justicia, cuya vinculación moral impide que sea objeto de negociación. Por lo demás, como ya quedó antes indicado, el consenso entreverado se dirige únicamente a las doctrinas «razonables», esto es, aquellas que de modo implícito admiten las condiciones intersubjetivas del discurso público liberal ${ }^{12}$, como ocurre en el diálogo del model case antes descrito. Por todo lo cual Rawls rechaza la acusación de utopismo sobre su teoría del consenso entreverado aunque recurre a un diseño histórico imaginario, pero plausible, del avance sostenido de su teoría liberal de la justicia: «apenas comiencen los ciudadanos a apreciar lo que ofrece una concepción liberal,

\footnotetext{
${ }^{10}$ PL 144.

${ }^{11}$ PL 147.

${ }^{12}$ PL 150.
} 
desplegarán respecto a la misma una lealtad que no hará más que fortalecerse con el tiempo» ${ }^{13}$.

Como puede apreciarse, la teoría rawlsiana del consenso entreverado, aun cuando pudiera aplicarse al diálogo transcultural sobre los derechos humanos, habría de ser modificada también fuertemente en su diseño para poder ser aceptado como un procedimiento imparcial para tal diálogo, dado su actual sesgo asimilacionista simple. Pues, aun concediendo que la teoría liberal de los derechos humanos ha quedado satisfactoriamente confirmada en la posición original y, por tanto, ha de ser presentada y defendida con fidelidad, ello no empece para que su presentación y defensa en el diálogo transcultural haya de hacerse con espíritu abierto y se someta a la indispensable traducción categorial, que posibilita no sólo el diálogo real, sino también el probable enriquecimiento mutuo -aunque no simétrico- que de tal confrontación leal puede colegirse.

\section{c) Rawls y su versión minimalista en la aplicación de los derechos humanos}

Por lo demás, y en contraste con esta exigencia maximalista de justificación, Rawls se muestra minimalista en su exigencia de reconocimiento universal de los derechos humanos. Tal posición minimalista puede ser acertada, en principio, pero habrá de justificar con mayor rigor y precisión la selección que parece efectuar de derechos humanos básicos y menos básicos, aunque entre los últimos sólo cite expresamente dos casos: la libertad de pensamiento y de asociación que puede ser no tan igualitaria en las sociedades jerárquicas, aunque un grado mínimo de tales libertades sea siempre exigido.

En efecto, Rawls comienza por caracterizar los derechos humanos como independientes de toda doctrina moral o filosófica comprehensiva, incluyendo la misma doctrina liberal. Esta caracterización le parece esencial para el objetivo de evitar que las sociedades jerárquicas los rechacen en cuanto «liberales o democráticos, o de algún modo distintivos de la tradición política occidental, que resultan perjudiciales para otras culturas» ${ }^{14}$. Por tanto han de enfocarse como «derechos humanos básicos que expresan una normativa mínima de instituciones políticas bien-ordenadas para todos los pueblos», en cuanto pertenecientes por derecho propio «a una sociedad política justa de pueblos». Es decir, los derechos humanos básicos se plantean en las sociedades democráticas y en las sociedades jerárquicas bien-ordenadas, pero con tal vigencia que una violación sistemática de tales derechos

\footnotetext{
${ }^{13}$ PL 158-160.

${ }^{14}$ LP 68.
} 
es incompatible con tal estatuto de sociedad liberal o jerárquica. Lo que, al tratarse de una normativa mínima, obliga, según Rawls, a presentar una versión «muy débil» (quite weak) de los mismos ${ }^{15}$.

Por otra parte, la forma debilitada de su enumeración no empece para nada la positivación de los derechos humanos en cuanto obligaciones vinculantes tanto para los ciudadanos como para los jueces y funcionarios. Y seguidamente explicita la lista mínima de derechos humanos a aceptar por los ciudadanos de tales sociedades: «derecho a la vida y seguridad, a propiedad personal, y los elementos del Estado de Derecho (the Rule of Law), así como el derecho a cierta libertad de conciencia y libertad de asociación, y el derecho a emigrar» ${ }^{16}$. Para la debida positivación de tales derechos y obligaciones bastan dos requisitos igualmente mínimos: una concepción común apropiada de la justicia y buena fe por parte de los funcionarios implicados para explicar y justificar tal orden legal a sus destinatarios. No es precisa, en cambio, la idea liberal de los ciudadanos como personas libres e iguales, pues se trata de justificar «un régimen mínimamente decente», y de este modo los derechos humanos se presentan como «políticamente neutrales», sin vincularlos a la tradición liberal occidental.

Por lo demás, Rawls reconoce que existen muchas sociedades con tradiciones distintas al individualismo occidental, en las que los ciudadanos son primordialmente miembros de grupos tales como «comunidades, asociaciones o corporaciones» ${ }^{17}$. Pero piensa que tal diferencia no resulta insuperable con tal de que se trate de «estados jerárquicos bien-ordenados» con su correspondiente «jerarquía de órganos de consulta», pues de su misma naturaleza se sigue la observancia de tales derechos humanos mínimos, aunque sea como miembros de agrupaciones y no como ciudadanos ${ }^{18}$.

Sin duda los derechos humanos están llamados a jugar en el momento actual un papel especial en un «razonable derecho de los pueblos». Rawls recuerda al respecto los dos cambios profundos que se han producido en el derecho internacional público desde la Segunda Guerra Mundial, que implican un cambio histórico: en primer lugar, la guerra no es considerada ya como un medio admisible de política estatal, sino que únicamente se justifica en el caso de autodefensa; en segundo lugar, la soberanía interna del estado está ahora limitada, y una de las funciones de los derechos humanos es la especificación de los límites de tal soberanía interna. Por otra parte, los derechos humanos han de ser diferenciados claramente de los derechos constituciona-

${ }^{15} \mathrm{LP}$ ib.

${ }^{16}$ LP ib.

${ }^{17}$ LP 69.

${ }^{18}$ LP 70. 
les o de los derechos de la ciudadanía democrática u otras clases de derechos individualistas o asociativos. Rawls los caracteriza como «una clase especial de derechos de aplicación universal y apenas controvertidos en su intención general» ${ }^{19}$. Pero significativamente Rawls distingue en nota derechos humanos de dos tipos: $1^{\circ}$, derechos humanos «propiamente» dichos, como los especificados por los artículos tres al dieciocho de la Declaración Universal de 1948, aun cuando subsistan algunas cuestiones de interpretación; además, otros derechos humanos que son «implicaciones» obvias de tales derechos, como son los casos extremos de genocidio y de «apartheid»; $2^{\circ}$, en otros casos, como el especificado por el primer artículo de dicha Declaración («Todos los seres humanos han nacido libres e iguales en dignidad y en derechos. Están dotados de razón y de conciencia y deben actuar mutuamente con espíritu de fraternidad») se trata más bien de fijar «aspiraciones liberales»; y en el caso de los artículos 22 y 23 presuponen instituciones específicas liberales (seguridad social y derecho a igual paga por igual trabajo, respectivamente). En definitiva, los derechos humanos han de cumplir, según Rawls, un triple papel: a) son condición necesaria para la legitimidad de un régimen y para la decencia de su ordenamiento legal; b) su vigencia basta para excluir toda intervención violenta justificada por parte de otros países, tal como mediante sanción económica o, menos todavía, mediante fuerza militar; c) por último, marcan un límite al pluralismo entre los pueblos ${ }^{20}$.

\section{d) EI cumplimiento efectivo de los derechos humanos}

Otra cosa es, claro está, plantear la cuestión del cumplimiento efectivo de los derechos humanos desde el enfoque «no-ideal de la teoría». Este planteamiento realista ha de tener en cuenta que las condiciones de nuestro mundo son «altamente no-ideales», con grandes injusticias y todo tipo de males sociales. Desde este enfoque no-ideal ha de plantearse una realización «paso por paso» de la teoría ideal, teniendo en cuenta que las políticas concretas hacia aquel objetivo sean, a la vez, posibles, efectivas y moralmente permisibles. Rawls las define como «cuestiones de transición»: hay que partir, en todo caso, del statu quo para buscar y encontrar caminos apropiados para el fin perseguido.

Pero hay que distinguir, según Rawls, dos tipos de incumplimiento de los derechos humanos: a) se da incumplimiento porque el régimen político se niega a reconocer un «razonable derecho de los pueblos»; tales estados pueden denominarse «forajidos» (fuera de la ley); b) se da incumplimiento a causa de las condiciones desfavorables para su aplicación en determina-

${ }^{19}$ LP ib.

${ }^{20}$ LP 71. 
dos países por circunstancias históricas, sociales y económicas, que hacen muy difícil o imposible el establecimiento de un «régimen bien-ordenado», sea éste liberal o jerárquico ${ }^{21}$.

Obviamente, las relaciones internacionales de los países «bien-ordenados» han de ser muy diferentes con los estados forajidos que con aquellos otros que han de luchar contra circunstancias desfavorables: con los primeros, sobre todo si son expansionistas, no cabe más que un modus vivendi, aunque haya que distinguir siempre a los gobernantes y élites del pueblo al que tienen sometido. Ahora bien, no es legítima la intervención armada contra tales regímenes más que en defensa propia y, en casos graves, en defensa de las personas inocentes subyugadas y para proteger los derechos humanos. La autoprotección es el deber primordial, aunque también ha de procurarse por parte de los «países bien-ordenados» la promoción de los derechos humanos por medio de una política juiciosa que incluye el organizarse en «nuevas instituciones y prácticas» al modo de «un centro federativo y un foro público» para consensuar su opinión y su política respecto a los demás países, así como para denunciar a la opinión pública «las instituciones injustas y crueles de los regímenes opresivos y expansionistas». Esta crítica será tanto más eficaz cuanto que no pueda ser tachada de «meramente liberal u occidental». Asimismo han de tomarse medidas de presión indirecta contra tales regímenes, como la denegación de ayuda militar, económica o asistencial, a la vez que se les niega el acceso a las «prácticas cooperativas» propias de los estados «bien-ordenados» ${ }^{22}$.

La actitud ha de ser muy distinta con los países que se encuentran en circunstancias desfavorables. Rawls entiende que estos países carecen de las tradiciones políticas y culturales, así como del capital humano y de know-how, además de los recursos materiales y tecnológicos que «hacen posible el establecimiento de sociedades bien-ordenadas» ${ }^{23}$. En este caso la actitud hacia tales regímenes ha de ser la de máxima asistencia y colaboración. Pero no cree que pueda exigirse al caso la aplicación del «principio de la diferencia», como estiman Beitz ${ }^{24}$ y Pogge ${ }^{25}$, entre otros, ya que su justifica-

${ }^{21}$ LP 71-72.

${ }^{22}$ LP 73-74.

${ }^{23}$ LP 74.

${ }^{24}$ C. R. Beitz, Political Theory and International Relations. Princeton, NJ, Princeton U.P., 1979, 125-176. Beitz cita como antecesores de esta idea (y crítica sobre Rawls) a B. Barry, P. Danielson y T. M. Scanlon. Una discusión conjunta puede verse en R. Amdur, «Rawls'Theory of Justice: Domestic and International Perspectives». World Politics 29, no. 3 (abril 1977), 438-461.

${ }^{25}$ T. W. Pogge, «An egalitarian Law of Peoples». Philosophy \& Public Affairs, 23, 1994, 195-224. Pogge había hecho ya una primera presentación de su teoría en el cap. 6 de su Realizing Rawls. Ithaca, Cornell University Press 1989. 
ción está limitada «a la teoría ideal para las sociedades democráticas» $\mathrm{y}$, sobre todo, porque se trata de un principio liberal de justicia distributiva y, como tal, no tiene por qué ser reconocida su validez por las sociedades jerárquicas. La razón de fondo es que la metodología constructivista no permite transportar un principio validado para la «justicia doméstica» como si hubiera sido justificado para regular las desigualdades en una sociedad de pueblos, pues cada cuestión «ha de ser gobernada por sus principios propios y característicos». En cambio «el deber de asistir» a tales sociedades bajo condiciones desfavorables es una obligación no meramente liberal, sino que atañe a todos los países bien-ordenados. $\mathrm{Y}$ tal deber es tanto más apremiante cuanto que «las condiciones desfavorables» pueden ser corregidas mediantes la aplicación de las políticas apropiadas. La promoción de los derechos humanos en tales países mediante prácticas asistenciales le parece la vía más apropiada para moderar, aunque sea lentamente, la corrupción de las élites, a la vez que estimula la aparición de una «cultura pública política» y la enraíza en la estructura social fundamental» ${ }^{26}$.

\section{e) Rawls entre el maximalismo y el minimalismo}

Es ahora el momento de precisar mi caracterización de Rawls como maximalista, al aplicar a las sociedades jerárquicas el procedimiento de validación que significa la posición original, y de minimalista en su aplicación universal de los derechos humanos. Respecto a su maximalismo me parece claro que la desautorización por parte de Rawls a Beitz y Pogge, quienes aplican su principio de la diferencia como integrante del derecho de los pueblos, sin darse cuenta de que se trata de un principio validado únicamente para las sociedades domésticas liberales mediante la metodología constructivista, le es plenamente aplicable al mismo Rawls y por las mismas razones, dado que la metodología constructivista parte necesariamente de unas convicciones compartidas que no se dan en las sociedades jerárquicas según la descripción de las misma que hace Rawls. Me parece ajustada su apreciación de que el principio distributivo de la diferencia, al ser un principio exclusivamente liberal, no es aplicable a las sociedades que se encuentran en condiciones desfavorables respecto a la implantación y el cumplimiento de los derechos humanos. Pero mi conclusión extiende el mismo principio y por las mismas razones a las sociedades jerárquicas.

No estoy de acuerdo, en cambio, que las relaciones internacionales entre las sociedades democráticas liberales y de los demás pueblos se rijan adecuadamente mediante el principio asistencial incorporado al derecho de los pueblos. En primer lugar, porque del hecho de que no pueda justificar-

${ }^{26}$ LP 75-77. 
se la vigencia internacional del principio de justicia distributiva entre todos los pueblos, no se sigue de que tal principio no pueda serle exigido a las democracias liberales en sus relaciones internacionales con los demás pueblos, porque el principio ha sido validado para ellas; pero tal validez no tiene por qué circunscribirse a las relaciones domésticas internas de cada democracia, y a las de las democracias entre sí, ya que aquella validación no depende de la reciprocidad de las sociedades no democráticas (que, ciertamente, se exige para el derecho internacional público). Lo justo (moral y democráticamente) sería, pues, que las democracias occidentales asumieran de modo inequívoco y sin más dilaciones la recomendación de la ONU para que destinen el $0,7 \%$ de su producto interior bruto a la ayuda a los países del Tercer Mundo. Incluso habría que estudiar seriamente la propuesta de W. T. Pogge ${ }^{27}$ para crear una «Tasa Global de Recursos», equivalente al $1 \%$ del valor del consumo, constituyendo un fondo de compensación a la escasez de recursos naturales y en previsión de las necesidades de las generaciones futuras de todo el mundo.

En segundo lugar, porque Rawls sigue manteniendo en 1993 la misma visión ya caduca que mantenía en 1971 (A theory of Justice) de los estados-naciones como unidades autosuficientes y soberanas, que sólo secundariamente tuvieran que relacionarse con los demás pues, como señala $\mathrm{Beitz}^{28}$, los estados-naciones actuales cumplen el criterio rawlsiano de ser unidades cooperativas a una escala globalizada en la que los límites territoriales son cada vez más difusos no sólo en las relaciones comerciales y culturales, sino también en las políticas, jurídicas y morales, por lo que el criterio tradicional que todavía mantiene Rawls de la ayuda mutua en situaciones excepcionales ha quedado claramente obsoleto.

$\mathrm{Su}$ minimalismo, en cambio, al desprender incoativamente los derechos humanos de su envoltura liberal, y al hacer una selección restringida de los mismos que incluya sólo los derechos humanos verdaderamente fundamentales, me parece acertada, por discutible que sea tal selección, en especial al admitir una cierta restricción de la libertad de pensamiento y de asociación en las sociedades jerárquicas; en efecto, una versión reducida de ambas libertades sólo podría aceptarse temporalmente, y ello si cumple uno de los criterios aplicados por el mismo Rawls en otro lugar: si «una libertad menos amplia puede favorecer el sistema total de libertad compartido por to-

${ }^{27}$ T.W. Pogge, loc.cit. en nota 23.

${ }^{28}$ C.R. Beitz, op. cit. en nota 22, pp. 127-176. Es de notar que Rawls (LP nota 52, 228-9) afirma expresamente que coincide con los objetivos de Beitz, pero que tales objetivos se pueden obtener por el principio asistencial, «sin necesidad del principio liberal de justicia distributiva». 
dos; algo menos que igual libertad puede ser aceptable para aquellos con menor libertad» $»^{29}$. Pero, en todo caso, Rawls se queda demasiado corto, dado que la formulación de los derechos humanos ha de presentarse traducida a las diversas condiciones socioculturales de los pueblos como condición indispensable tanto de su adecuada expresión como para su aceptación universalista por las sociedades no liberales.

Obviamente, los derechos humanos fundamentales no dependen de las concepciones liberales democráticas, sino que su valor intrínseco, desprovisto del ropaje liberal, se alcanza básicamente a través del desarrollo de los valores universales de la naturaleza humana, con la sola condición de que tal crecimiento no sea impedido por imposiciones o desviaciones ideológicas o corruptas. Lo normal es, por tanto, que se alcancen valores humanos equivalentes en diferentes culturas. Y sobre tales valores habrá de realizarse la aludida transposición intercultural de los derechos humanos liberales. Sin que pueda negarse, por lo demás, que la conquista revolucionaria de los derechos humanos constituyen la gran aportación de Occidente a la humanidad.

Por lo demás, estoy persuadido de que la mejor estrategia para la promoción y universalización de los derechos humanos no es directa, sino indirecta, ya que su aceptación progresiva está estrechamente vinculada -y hasta dependiente- a la promoción y universalización de la democracia en el mundo, que también habrá de hacerse desprendida de su envoltura liberal y traducida tanto categorial como institucional mente a las características socioculturales de cada país, de tal modo que se obtenga, a la vez, una transición democrática auténtica y adaptada de tal modo que, al igual que sucedía con la universalización de los derechos humanos, no sea preciso «occidentalizar» a los destinatarios de la misma. Y ello por exigencias de autenticidad, que en este caso coinciden en gran manera con las exigencias estratégicas: ni la democracia ni los derechos humanos podrán ser aceptados en su ropaje occidental-liberal. Obviamente, la universalización de los derechos humanos es mucho más prioritaria que la del régimen democrático; pero, en la práctica, ambas universalizaciones habrán de ser paralelas puesto que ambas son interdependientes: el riguroso cumplimiento de los derechos humanos es el más claro criterio de democratización, ya que, como señala justamente Rawls, marcan los límites de la tolerancia y del pluralismo, y, a la vez, tal objetivo sólo será posible en una democracia verdadera.

${ }^{29}$ J. Rawls, A Theory of Justice. Cambridge, Mass., Harvard University Press, 1971, 302. 
DOXA 21-II (1998) 